\title{
Demonstration of Negative Impedance Conversion for Bandwidth Extension in VLC
}

\author{
Amany Kassem and Izzat Darwazeh \\ Department of Electronic and Electrical Engineering, University College London, London, United Kingdom \\ Email: amany.kassem.15@ucl.ac.uk, i.darwazeh@ucl.ac.uk
}

\begin{abstract}
This work proposes and demonstrates the utility of a negative impedance converter (NIC) circuit, based on a common collector (CC) amplifier, for the generation of negative capacitance. The design principle of the proposed NIC is introduced, then a negative capacitance equals $-200 \mathrm{pF}$ is demonstrated using discrete devices constructed on a printed circuit board (PCB). The designed NIC is applied for the bandwidth extension of LEDs to enhance the achievable data rates in visible light communication (VLC) systems. The paper includes analytical derivations of the obtained negative capacitance as a function of circuit parameters and verifies this by both simulation and experimentally. Measurements show significant bandwidth extension by neutralising the bandwidth-limiting effect of the LED diffusion capacitance through the introduction of a parallel negative capacitance.
\end{abstract}

Index Terms-Negative impedance converters, negative capacitance, light emitting diodes, visible light communication.

\section{INTRODUCTION}

Negative impedance can be realised using active networks known as negative impedance converters (NICs). NIC is defined as a two port network that presents negative impedance at one port when terminated by the corresponding load impedance at the other port [1-3]. NICs can be designed to generate either negative resistance, capacitance or inductance depending on the terminating load impedance.

Due to their attractive capability of producing unconventional impedances, NIC networks are widely used to improve the performance of various applications. For example, enhancing the bandwidth of amplifiers by neutralising bandwidthlimiting capacitances [4-7], improving the quality factor of active filters by compensating for resistive losses [8], improving the bandwidth of microwave antennas [9] and broadband matching of small electrical antennas to overcome the gainbandwidth restriction [10].

There are various forms to devise NICs; originally based on amplifiers employing BJTs as demonstrated, in 1953, by Linvill [2] and later by using cross-coupled MOSFETs structures as in $[4,5,11]$. Another possibility is employing operational amplifiers (op-amps) as in [12]. Although, the adoption of the aforementioned NIC architectures has proven to be versatile and effective, yet they mostly suffer from a number of drawbacks that limit their utility to low frequency applications. Mainly, NICs are inherently unstable due to the presence of positive feedback and they may have limited operating range, caused by the non-ideal characteristics of the active devices, such as the finite input impedance and non-zero output impedance [13].
This work proposes a new application of NICs; that is the bandwidth extension of light emitting diodes (LEDs) when used, for example, in high capacity visible light communication (VLC) systems. It is well-known that the modulation bandwidth of commercially available LEDs is typically limited to a few $\mathrm{MHz}$, which severely inhibits transmission rates [14]. The bandwidth is mostly limited by the carrier lifetime and the RC time constant set by the LED dynamic resistance $\left(r_{d}\right)$ and the diffusion capacitance $\left(C_{d}\right)$ [14,15]. Following from a recent study in [16], this work proposes a new LED bandwidth extension technique by means of introducing a synthesized negative capacitance in parallel with the bandwidth-limiting $C_{d}$, in a topology with similarities to that outlined in [7]. Full circuit design is detailed and shown to achieve bandwidth extension by generating negative capacitance using a specially designed NIC, which capitalizes on the distinct characteristics of the LED. Significant bandwidth extension is demonstrated without trading optical power, as opposed to other LED bandwidth extension techniques in [15, 17].

In this work, we develop a current inversion NIC circuit based on applying positive feedback to a common collector amplifier. We experimentally show that a stable, wide-band negative capacitance can be realised. The circuit performance is examined while varying some design parameters such as NIC bias current. We propose for the first time, the application of NIC to extend the bandwidth of LEDs for VLC systems.

\section{DESIGN OF THE PROPOSED NIC CIRCUIT}

\section{A. Negative impedance converter design}

According to [1], NICs are based on amplifiers that employ positive feedback, whereas the load to be inverted is connected in the feedback loop. A NIC where the input and output voltage are of a opposite polarity is termed a voltage inversion NIC and conversely one where the current is inverted is a current inversion NIC.

Fig. 1 illustrates a practical implementation of a current inversion NIC, constructed as a common collector stage (CC) $Q 1$ and a common base (CB) stage $Q 2$. The positive feedback is formed by connecting the output and input of $\mathrm{CC}$ stage through the capacitance $C_{c}$ and the low input impedance CB stage. Ideally, the capacitance $C_{c}$ will be negated at the input, where $C_{i n}=-C_{c}$. This $C_{i n}$ would appear as a shunt element and can be used advantageously where shunt capacitance neutralization is beneficial. 


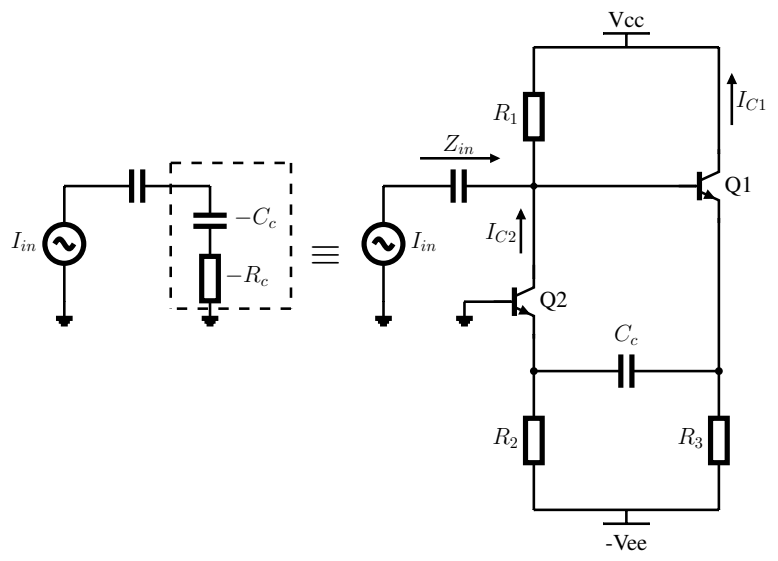

Fig. 1. Practical implementation of a current inversion NIC

The NIC input impedance is derived by applying nodal analysis to the corresponding simplified small signal model of the NIC circuit in Fig. 1. This analysis makes reasonable and practical simplifying assumptions of ideal transistors $Q 1$ and $Q 2$, with negligible internal capacitances and large bias resistors, resulting in negligible NIC loading. Leading to a compact equation for $Z_{i n}$, and a passive equivalent circuit emulating the NIC input impedance. Hence, the derived NIC input impedance, resistance and reactance are given by:

$$
\begin{gathered}
Z_{i n} \approx \frac{g_{m 2}\left(1+g_{m 1} r_{\pi 1}\right)+j \omega C_{c}\left(1+g_{m 1} r_{\pi 1}+g_{m 2} r_{\pi 1}\right)}{j \omega c_{c} r_{\pi 1} g_{m 1} g_{m 2}} \\
R_{c} \approx-\frac{g_{m 1}+g_{m 2}}{g_{m 1} g_{m 2}}, \quad X_{c}=\frac{1}{\omega C_{c}}
\end{gathered}
$$

By inspecting (2), it can be concluded that the proposed NIC presents an impedance combination equals a negative capacitance $-C_{c}$ in series with negative resistance $-R_{c}$. The magnitude of the negative capacitance equals the terminating capacitance $C_{c}$ and $-R_{c}$ is a function of the transconductances $g_{m 1}$ and $g_{m 2}$ of $Q_{1}$ and $Q_{2}$. The NIC impedance equivalent circuit shown by Fig. 1 (dashed box).

In general NICs are prone to instability due to the nature of their operation, which incorporates positive feedback [2]. Therefore, to prevent NICs from oscillating, it is necessary to place a stabilizing element at the input. The nature of the stabilizing element is determined by load being converted. For instance, if the NIC is negating a load resistor $R_{L}$ then a stabilizing input resistance $R_{i n}$ is required so that the net resistance remains positive $R_{n e t}=R_{i n}-R_{L}>0$. The necessary conditions for NIC stability has been extensively studied in $[10,18]$, which are mainly based on the Nyquist stability criterion to ensure the network function does not have any poles in the right hand side of the s-plane. Without exception, the proposed NIC requires a stabilizing input element. A stabilizing shunt input capacitance $C_{i n}$ is used to ensure the net input capacitance always remains positive $\left(C_{n e t}=C_{i n}-C_{c}>0\right)$. Moreover, it is possible to further enhance the stability by adding base stopper resistor at the base of $Q 1$ shown in Fig. 1 [19].

\section{B. Passive equivalent circuit analysis}

This section investigates the application of the proposed NIC to extend the bandwidth of LEDs. We examine the LED impedance characteristics independently and when connected to the NIC by analysing simplified passive equivalent circuits. The impedance of LEDs is relevant to this study as was shown in [15], when driven by a current source the impedance follow the same profile as the current through the LED $i_{d}$.

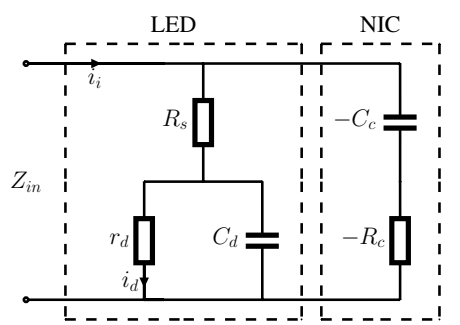

Fig. 2. Simplified LED equivalent in parallel with synthesised negative capacitance

Fig. 2 illustrates a passive equivalent circuit consisting of the well-known LED model in parallel with the equivalent circuit of the proposed NIC. The simplified LED model consists of the diffusion capacitance $C_{d}$, the dynamic resistance $r_{d}$ and the Ohmic contact resistance $R_{s}$. By analysing the stand-alone LED model in Fig. 2 the LED impedance is given by:

$$
Z_{\text {in }}=R_{s}+\frac{r_{d}}{1+j \omega r_{d} C_{d}}
$$

The LED exhibits a first order low pass filter where the bandwidth is dictated by a time constant equals $r_{d} C_{d}$. The LED bandwidth can be potentially extended by ameliorating the bandwidth-limiting effect of $C_{d}$ through introducing a parallel negative capacitance $-C_{c}$, thereby reducing the net capacitance dictating the bandwidth. Ideally, the bandwidth can be infinitely increased if $\left|C_{c}\right|=C_{d}$, so the LED bandwidth becomes:

$$
f_{3 \mathrm{~dB}}=\frac{1}{2 \pi r_{d}\left(C_{d}-C_{c}\right)}
$$

Nevertheless, as previously shown it is not possible to realise pure negative capacitance, yet the NIC impedance consists of $-C_{c}$ in series with $-R_{c}$. Therefore, analysing the LED impedance when connected to the NIC in Fig. 2 yields the impedance given by (5).

From (5), it is clear that the DC LED resistance remains the same as in (3) equals to $R_{s}+r_{d}$. Moreover, the LED impedance function has two real zeros (6) and either a real pole or a complex conjugate pole. In cases were $R_{s}=\left|R_{c}\right|$, then the second order term cancels, the transfer function has just one real pole as in (4).

$$
\omega_{z 1}=\frac{R_{s}+r_{d}}{r_{d} R_{s} C_{d}}, \omega_{z 2}=\frac{1}{R_{c} C_{c}}
$$

$$
Z_{\text {in }}=\frac{\left(r_{d} R_{s} C_{s} s+r_{d}+R_{s}\right)\left(R_{c} C_{c} s+1\right)}{\left(r_{d} C_{d} C_{c}\left(R_{s}-R_{c}\right)\right) s^{2}+\left(r_{d}\left(C_{d}-C_{c}\right)-C_{c}\left(R_{s}-R_{c}\right)\right) s+1}
$$


However, in cases where $R_{s} \neq\left|R_{c}\right|$, then the transfer function has a complex pole given by:

$$
p_{1,2}=-\frac{\omega_{n}}{2 Q}+j \omega_{n} \sqrt{1-Q^{2}}
$$

where $\omega_{n}$ is the complex conjugate pole natural frequency and $Q$ is the quality factor, expressed by:

$$
\omega_{n}=\frac{1}{\sqrt{r_{d} C_{d} C_{c}\left(R_{s}-R_{c}\right)}}, Q=\frac{\sqrt{r_{d} C_{d} C_{c}\left(R_{s}-R_{c}\right)}}{r_{d}\left(C_{d}-C_{c}\right)-C_{c}\left(R_{s}-R_{c}\right)}
$$

Hence, by inspecting (5), three key conclusions can be made: $(i)$, the LED bandwidth is no longer determined by $C_{d}$ and $r_{d}$ only. Moreover, the value of $R_{c}$ in (2) can be designed so that $R_{s}=\left|R_{c}\right|$ and hence, the bandwidth is given by (4) and is determined by a time constant equals $r_{d}\left(C_{d}-C_{c}\right)$. While, complete neutralisation of $C_{d}$ is not possible, as it would compromise the NIC stability yet optimisation is feasible. In cases where $R_{s} \neq\left|R_{c}\right|$ the system has a complex pole; where $\omega_{n}$ increases as $-C_{c}$ is increased (becomes more negative). As $\omega_{n}$ increases, it shifts the frequency of $p_{1,2}$ to higher frequencies, thus extending the LED bandwidth. (ii) The zero frequency $\omega_{z}$ expressed in (7) is a function of $R_{c}$ and $C_{c}$.

Finally, (iii), the LED DC resistance is not reduced when connected to the NIC, which means there is no resistive loading. Hence, there will be no LED output power loss. However, this is dependent on the exact design of the NIC. In case of the circuit in Fig. 1, there will be additional loading due to the bias resistor $R_{1}$. However, if $R_{1}>>R_{s}+r_{d}$, such loading may be neglected. Hence, the proposed LED extension technique does not impose a bandwidth-power trade-off and the bandwidth extension does not come at the expense of loss in optical power as with techniques reported in [15].

\section{Demonstration OF THE PROPOSED NIC}

We have designed, built and tested a stable negative capacitance using the NIC circuit shown in Fig. 1. Fig. 3 shows the proposed NIC fabricated on a FR4 double-sided printed circuit board (PCB). The PCB is populated using silicon NPN transistors BFR193 with transition frequency $f_{T}=8 \mathrm{GHz}$ [20], and assortment of surface mount devices (SMD) including biasing resistors from $R 1$ to $R 3$.

The scattering parameters of the fabricated NIC are measured using a vector network analyzer (VNA), which are then converted into its corresponding impedance parameters to obtain the generated negative capacitance.

The NIC performance is tested by constructing the setup shown in Fig. 4. Where, a shunt stabilizing capacitance $C_{i n}$ is placed at the NIC input and the NIC is terminated with an arbitrary chosen value of $C_{c}$. This $C_{c}$ appears at the input in parallel with $C_{i n}$ as a combination of $-C_{c}$ in series with $-R_{c}$. Hence, resulting in partial neutralization of $C_{i n}$ and therefore, reduction in the net capacitance $C_{n e t}$ seen by VNA port 1 .

The value of $-R_{c}$ dictates the quality at which $-C_{c}$ neutralizes $C_{i n}$. Ideally, $-R_{c}$ should be zero to obtain pure negative capacitance. However, this is not achievable in practice since $-R_{c}$ is a function of the NIC bias currents $I_{C 1}$ and $I_{C 2}$ as given by (2). Nevertheless, increasing the bias currents would reduce $-R_{c}$, hence, enhancing the quality at which $C_{i n}$ is neutralized.

The effect of the inevitable $-R_{c}$ on the quality of neutralizing $C_{i n}$ is examined by measuring the reflection co-efficient (S11) of the NIC for different bias currents. Whereas, $I_{C 1}$ and $I_{C 2}$ are varied by changing the values of $R_{4}$ and $R_{5}$, whilst maintaining the appropriate bias conditions. The bias currents are constantly maintained so that the value of $I_{C 1}=I_{C 2}=I_{C}$. Subsequently, S11 values are converted into its corresponding $\mathrm{Z} 11$ values with the net capacitance $C_{n e t}$ obtained from the reactive component of the $\Im\left(Z_{11}\right)$ using:

$$
C_{n e t}=\frac{1}{2 \pi f \Im\left(Z_{11}\right)}
$$

Fig. 5 shows the measured and simulated at different bias currents. Whereas, $C_{i n}=600 \mathrm{pF}$ and $C_{c}=200 \mathrm{pF}$, hence, ideally $C_{n e t}=400 \mathrm{pF}$. Initially, we take a measurement of stabilizing capacitance $C_{i n}$ while connected to the NIC, yet with no compensating capacitor $C_{c}$ (NIC open circuit). This measurement taken to serve as a comparator to cases where the NIC is terminated by $C_{c}$ for different $I_{C}$, is the top curve in Fig. 5.

It can be seen that the de-embedded value of $C_{i n}$ starts at almost $640 \mathrm{pF}$ and continues to increase as function of frequency. This frequency dependent behaviour has been verified in simulation by employing SMD capacitor model provided by the manufacturer. Moreover, by accounting for the PCB trace connecting the VNA input and $C_{i n}$, which introduces significant series inductance and hence, the increasing value of $C_{i n}$. By looking at cases where the NIC is terminated by $C_{c}=200 \mathrm{pF}$ for the three different $I_{C}$, it is clear that in all cases there is reduction in capacitance in comparison to $C_{i n}$. Higher values of $I_{C}$ yields a less frequency dependent $C_{n e t}$, which is due to the reduction of $-R_{c}$.

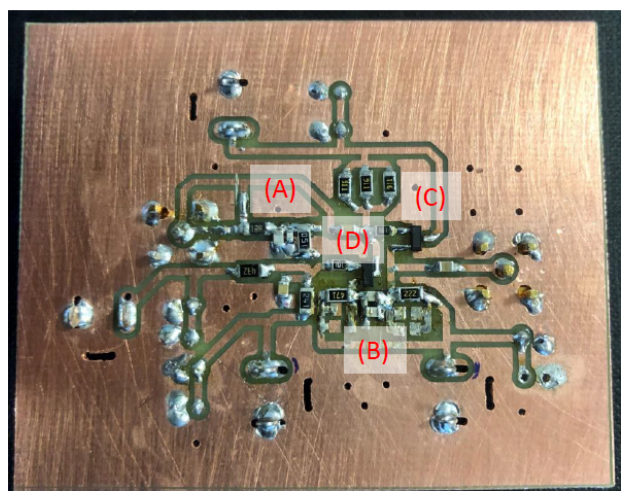

Fig. 3. Fabricated NIC PCB (A) LED equivalent model (B) NIC compensating capacitor $C_{c}$ (C) NIC common collector stage (D) NIC common base stage

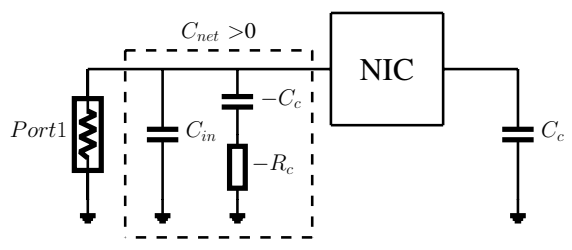

Fig. 4. Test setup 




Fig. 5. Measured and simulated $C_{n e t}$ at the NIC input under different $I_{C}$



Fig. 6. Measured and simulated negative capacitance under different $I_{C}$

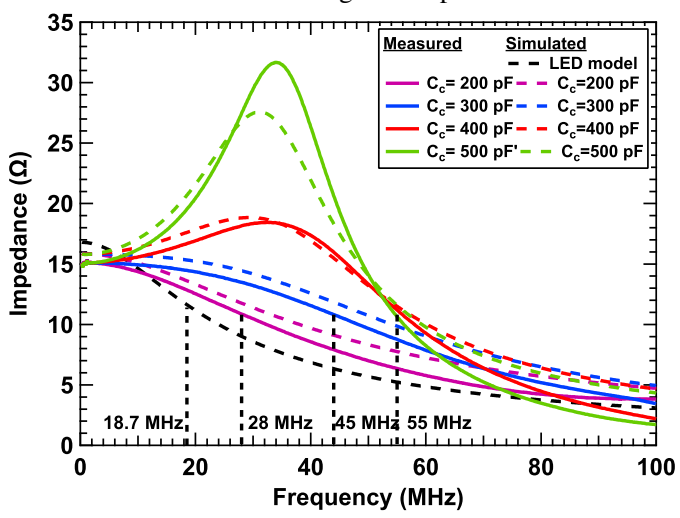

Fig. 7. LED impedance response when connected to the NIC to different $C_{c}$ under $I_{C}=24 \mathrm{~mA}$

Fig.6 shows the negative capacitance generated by the NIC, which is obtained by subtracting $C_{n e t}$ from the value of $C_{i n}$. It can be clearly seen that increasing $I_{C}$ provides a less varying value of $C_{c}$. This is because increasing $I_{C}$ also increases the transconductances $g_{m 1}$ and $g_{m 2}$, which has the direct result of reducing $-R_{c}$ (becomes less negative). Hence, obtaining a flatter value of $C_{c}$ across frequency.

\section{APPLICATION OF NIC LEDS BANDWIDTH EXTENSION}

In this section, we demonstrate the effect of compensating for the bandwidth-limiting LED diffusion capacitance by exploiting NICs to generate parallel negative capacitance. For consistent results and fair comparison, we base both our simulations and measurements on the verified LED equivalent model in [21] with the characteristic values derived at $2 \mathrm{~mA}$ drive current are $C_{d} \approx 600 \mathrm{pF}, r_{d}=15 \Omega$ and $R_{s}=1.8 \Omega$. Using a similar test setup as in Fig. 3, with $C_{i n}$ replaced with the LED equivalent model.

Fig. 7 compares the LED impedance to four cases at which the LED is connected to the NIC while terminated by $C_{c}$ values in the range of $200 \mathrm{pF}$ to $500 \mathrm{pF}$ at $I_{C}=24 \mathrm{~mA}$. The NIC is biased at $24 \mathrm{~mA}$ to give maximise bandwidth extension.

As reported in [21], the LED equivalent model is predicted to have a raw bandwidth of $18.7 \mathrm{MHz}$ and $\mathrm{DC}$ resistance approximately equals $17 \Omega$. When $C_{c}=200 \mathrm{pF}$, the NIC generates $-C_{c}=-200 \mathrm{pF}$ in parallel with $C_{d}$, reducing the overall capacitance and resulting in improvement in the LED operating bandwidth of approximately $50 \%$. Moreover, looking at the DC resistance value it can be seen that the NIC results in very slight reduction in the LED resistance; less than $2 \Omega(10 \%)$. This slight reduction is due to the resistive loading caused by $R_{1}$ in Fig. 1 . As $C_{c}$ is further increased to $300 \mathrm{pF}$, the LED bandwidth is extended by $140 \%$, while maintaining the same DC resistance as $C_{c}=200 \mathrm{pF}$. As $C_{c}$ continues to increase to $400 \mathrm{pF}$, we observe that the LED bandwidth is improved by approximately $200 \%$ and the DC resistance remains as in the other cases. Clearly, the slight reduction in the LED impedance (optical power) is negligible in comparison to the significant gains in bandwidth.

We also note that the impedance profile becomes more flat with a slight peak in comparison to the over-damped responses of $C_{c}=200 \mathrm{pF}$ and $300 \mathrm{pF}$. The reason for this is that the frequency of the zero in (6) is a function of $C_{c}$. Hence, as $C_{c}$ continues to increase, the zero gradually shifts to lower frequencies and becomes closer to the complex pole. Hence, this would result in increased peaking of the response as $C_{c}$ is increased. This effect is confirmed by further increasing $C_{c}$ to $500 \mathrm{pF}$, where we observe no improvement in the NIC bandwidth and significant increase in the peak amplitude. This is because the zero is now at a lower frequency than that of the complex pole.

\section{CONClusion}

In this paper, we report the design and verification of a negative impedance converter circuit for the generation of negative capacitance to cancel the effects of LED diffusion capacitance and extend bandwidth. We mathematically analyze the proposed NIC and describe the design optimisations necessary to realise a stable high quality shunt negative capacitance. The NIC performance is evaluated through the measurement of the scattering parameters of a circuit constructed using discrete components on a PCB. Measurements show that stable high quality negative capacitance is achieved, which suggests it to be a good candidate for the equalisation of the bandwidthlimiting diffusion capacitance associated with LEDs for high capacity visible light communication systems. We demonstrate the utility of the proposed NIC by applying it to an LED equivalent model, which yields substantial bandwidth extension without trading off the LED optical power. 


\section{REFERENCES}

[1] J. L. Merrill, "Theory of the negative impedance converter," The Bell System Technical Journal, 1951.

[2] J. G. Linvill, "Transistor Negative-Impedance Converters," Proceedings of the IRE, 1953.

[3] A. Larky, "Negative-impedance converters," IRE Transactions on Circuit Theory, 1957.

[4] J. Han, K. Yoo, D. Lee, K. Park, W. Oh, and S. M. Park, "A lowpower Gigabit CMOS Limiting Amplifier Using Negative Impedance Compensation and Its Application," IEEE Transactions on Very Large Scale Integration (VLSI) Systems, 2012.

[5] S. Galal and B. Razavi, "10-Gb/s limiting amplifier and laser/modulator driver in $0.18-\mu \mathrm{m}$ CMOS technology," IEEE Journal of Solid-State Circuits, 2003.

[6] J. D. Drew, I. Z. Darwazeh, and B. Wilson, "Generalised model for prediction and synthesis of negative capacitance at microwave frequencies using common-gate MESFET," in 1996 26th European Microwave Conference, 1996.

[7] P. V. Golden and P. J. Mole, "Amplifiers with negative capacitance circuits," US 7,863,980 B, 2011.

[8] D. V. Kamat, P. V. A. Mohan, and K. G. Prabhu, "Active-RC filters using two-stage OTAs with and without feed-forward compensation," IET Circuits, Devices Systems, 2011.

[9] J. M. C. Covington, K. L. Smith, J. W. Shehan, V. S. Kshatri, T. P. Weldon, and R. S. Adams, "Measurement of a CMOS negative inductor for wideband non-foster metamaterials," in IEEE SOUTHEASTCON 2014, 2014.

[10] S. E. Sussman-Fort and R. M. Rudish, "Non-foster Impedance Matching of Electrically-Small Antennas," IEEE Transactions on Antennas and Propagation, 2009.

[11] B. Mrković and M. Ašenbrener, "The simple CMOS negative capacitance with improved frequency response," in 2012 Proceedings of the 35th International Convention MIPRO, 2012.

[12] A. N. Beal, J. N. Blakely, and N. J. Corron, "Extended-bandwidth Negative Impedance Converters by Nested Networks," IEEE Transactions on Circuits and Systems II: Express Briefs, 2018.

[13] Y. Shen and T. Chio, "Limitation of Negative Impedance Converter using Operational Amplifier for matching electrically small antenna," in 2013 IEEE Antennas and Propagation Society International Symposium (APSURSI), 2013.

[14] S. Rajbhandari, J. J. D. McKendry, J. Herrnsdorf, H. Chun, G. Faulkner, H. Haas, I. M. Watson, D. O'Brien, and M. D. Dawson, "A review of gallium nitride LEDs for multi-gigabit-per-second visible light data communications," Semiconductor Science and Technology, 2017.

[15] T. Odedeyi, P. A. Haigh, and I. Darwazeh, "Transmission Line Synthesis Approach to Extending the Bandwidth of LEDs for Visible Light Communication," in IEEE CSNDSP, 2018.

[16] A. Kassem and I. Z. Darwazeh, "Exploiting Negative Impedance Converters to Extend the Bandwdith of LEDs for Visible Light Communications," in IEEE International Conference on Electronics Circuits and Systems, 2019.

[17] H. L. Minh, D. O’Brien, G. Faulkner, L. Zeng, K. Lee, D. Jung, Y. Oh, and E. T. Won, "100-Mb/s NRZ Visible Light Communications Using a Postequalized White LED," IEEE Photon. Technol. Lett., 2009.

[18] D. Segovia-Vargas, J. Jimenez-Martin, A. Parra-Cerrada, F. AlbarracinVargas, E. Ugarte-Munoz, and V. Gonzalez, "Stability Analysis and Design of Negative Impedance Converters: Application to Circuit and Small Antennas," 2016.

[19] J. E. Green, R. Tozer, and J. P. R. David, "Stability in Small Signal Common Base Amplifiers," IEEE Transactions on Circuits and Systems I: Regular Papers, 2013.

[20] NPN Silicon RF Transistor, SIEMENS, Dec. 1996.

[21] W. N. Cheung, P. J. Edwards, and G. N. French, "Determination of LED equivalent circuits using network analyser measurements," in 1998 Conference on Optoelectronic and Microelectronic Materials and Devices. Proceedings (Cat. No.98EX140), 1998. 